\title{
LA VULNERABILIDAD COMPRENDIDA DESDE LA FILOSOFÍA, LA SOCIOLOGÍA Y EL DERECHO. DE LA NECESIDAD DE UN DIÁLOGO INTERDISCIPLINARIO
}

\author{
LAURENCE BURGORGUE-LARSEN ${ }^{1}$
}

RESUMEN: La noción de vulnerabilidad, ya recogida en varios estudios sociológicos y filosóficos, ofrece un nueva forma de (re)pensar la relación del ser humano con el Otro y con su sociedad. Por la importancia de la visión que ofrece, los juristas deben aproximarse a ella, pues brindará un nuevo campo de aplicación del derecho, no solamente a partir de las personas vulnerables, sino de la identificación de los contextos de vulneración. Los derechos humanos constituyen un espacio idóneo para el estudio de la vulnerabilidad, desde una visión interdisciplinaria.

PALABRAS CLAVE: vulnerabilidad, derechos humanos, igualdad, discriminación.

ABSTRACT: The notion of vulnerability, contained in several sociological and philosophical studies, offers a new approach about the relationship of humans with the Other and the society. From this view, the lawyers has a new field of application of law: not only regarding the vulnerable person, but identifying the contexts of vulnerability. The Human Rights are an appropiate area for the use of vulnerability, in interdisciplinary perspective.

KEY WORDS: vulnerability, human rights, equality, discrimination.

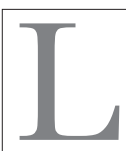

La vulnerabilidad, vista en esta perspectiva, introduce el tema de la relación entre los individuos y la sociedad en la cual viven. Cuestiona la "representación del ser humano"2 en el marco de la Cité, la Polis. Y si esta representación ha evolucionado

1 Profesora de la Escuela de Derecho de la Sorbonne. Instituto de investigaciones en derecho internacional y europeo. (IREDIES).

${ }^{2}$ Cffr. Genard, J. L., "Une réflexion sur l'anthropologie de la fragilité, de la vulnérabilité et de la souffrance", en Destins politiques de la souffrance, París, Érès, 2009, p. 28. 
profundamente desde hace aproximadamente veinte años es porque la sociedad se ha transfigurado. El modelo del Estado benefactor después de la guerra, el Estado social y protector, no existe más; el Welfare State ha vencido.

Frente a estos cambios estructurales en aspectos políticos, económicos, sociales y familiares, reveladores de esta desaparición del modelo social originado después de la Guerra, han aparecido nuevas formas de comprensión y de tratamiento de la relación entre el individuo y la frágil sociedad en la que se desenvuelve. Filósofos de un lado, sociólogos en el otro, se han enfocado con decisión en el estudio y en el análisis de nuevas formas de subjetividad contemporánea. Adieu a los análisis en términos de autonomía y de igualdad de derechos y vive a aquellos que enfatizan la dependencia en las relaciones y en la igualdad diferenciada, no universalista. La vulnerabilidad, por tanto, ha levantado el vuelo, ha triunfado en distintos campos de las disciplinas sociales: de la filosofía a la sociología, pasando por el derecho.

Son varios los movimientos filosóficos que han abordado, de manera más o menos directa, el concepto de vulnerabilidad. Lo han hecho para construir teorías que, al mismo tiempo, son explicativas sobre las nuevas relaciones de los individuos y la sociedad, y normativas, en el sentido que implican, necesariamente, repensar las características y las modalidades de la acción y del espacio públicos. De las aproximaciones hechas desde las capabilities (capacidades) del economista Amartya $\mathrm{Sen}^{3}$ y de la filósofa Martha Nussbaum, ${ }^{4}$ pasando por aquella de care expuesta en los Estados Unidos por Carol

3 Cfr. En versión original: Sen, Amartya, Inequality reexamined, Oxford, Clarendon Press, 1992; en francés: Repenser l'égalité, traducción de P. Chemla, París, Seuil, 2012, 320 p.

4 Cfr. Nusbbaum, Martha C., Creating Capabilities, The Human Development Approach, Cambridge, Harvard University Press, 2011, 337 p. 
Gilligan $^{5}$ o por Joan Tronto ${ }^{6}-\mathrm{y}$ desarrollada en Francia principalmente por Sandra Laugier ${ }^{-7}$ hasta llegar a la teoría del reconocimiento de Axel Honneth ${ }^{8}$ o al posicionamiento post-estructuralista de Judith Butler, ${ }^{9}$ los filósofos no han escatimado esfuerzos para tomar distancia de la filosofía moral y política encarnada por John Rawls en su famosa Teoría de la Justicia.$^{10}$ Los tiempos corresponden a los cambios epistemológicos: el "sujeto liberal", independiente, autónomo, responsable y autosuficiente, no aparece tan potente como lo era antes.

Estas recientes corrientes filosóficas - que se podría aventurar a considerarlas como subversivas, en tanto excluyen la aproximación

5 Cfr. Gilligan, C., In a Different Voice: Psychological Theory and Women's Development, Cambridge, Harvard University Press, 1982.

6 Cfr. En versión original: Tronto, JoAn, Moral Boundaries. A Political Argument for an Ethic of Care, Nueva York, Routledge, 1993; en francés: Un monde vulnérable. Pour une politique du Care, París, La Découverte, 2009, 240 p.

7 Cfr. Laugier, S., y Paperman, P., Le souci des autres. Ethique et politique du Care, París, Editions de l'EHESS, Raisons pratiques, 2006, 348 p. También Molinier, P., Laugir, S., y Paperman, P. (eds.), Qu'est-ce que le care? Souci des autres, sensibilité, responsabilité, París, Payot et Rivages, 2009, 302 p.

8 Cfr. Honneth, Axel, La lutte pour la reconnaissance, París, Cerf, 2000, 240 p. La versión original en alemán fue publicada en 1992. Emmanuelle Jouannet ha utilizado los trabajos del profesor alemán para pensar y teorizar la existencia de un derecho internacional del reconocimiento. Ver su obra Qu'est-ce qu'une société internationale juste? Le droit international entre développement et reconnaissance, París, Pedone, 2011,306 p.

9 Cfr. En edición original: ButLer, Judith, Excitable speech, Routledge; en francés: Le pouvoir des mots. Discours de haine et Politique du performatif, Traducción de C. Nordmann, París, Editions Amsterdam, 287 p.

10 Vid., la perspectiva contundente sobre varias de estas teorías en SuPIOT, Alain, "L’idée de justice sociale", en La Fustice sociale saisie par les juges en Europe, Burgorgue-Larsen, Laurence (ed.), París, Pedone, 2013, pp. 5-30. Aprovecha Supiot para presentar su propia idea al considerar: "La cuestión de la justicia no puede limitarse a la distribución de la riqueza producida o al reconocimiento de las identidades precedentes, pues, ello implica que cada uno se da cuenta de lo que es en lo que hace, para forjar su persona en un intento de trabajo. Un orden tiene que ser justo para dar la oportunidad a todos los seres humanos." 
clásica liberal- han terminado por tener una fuerte influencia en Francia. A título de ejemplo, se mencionará el ensayo de la filósofa Fabienne Brugère que propone - principalmente a la izquierda francesa- una política de sostenimiento o apoyo (soutien). ${ }^{11}$ Vinculando todas las aproximaciones filosóficas citadas en los párrafos anteriores, ${ }^{12}$ afirma que

[...] l'individu ne tient pas tout seul en société. Une politique de l'individu soucieuse d'égalité, qui vise le bien-être de tous les individus, doit pouvoir organiser des soutiens qui donnent à la fois des capacités et des possibilités d'agir à tout le monde, hors des statuts et des partages, et n'oublient pas les individus les plus vulnérables, ces 'individus négatifs' qui font l'épreuve du manque, de l'exclusion ou de l'invisibilité. ${ }^{13}$

También los sociólogos, por su lado, han transformado los fundamentos de sus análisis tradicionales para poder desmenuzar la

11 Cfr. Brugère, Fabienne, La politique de l'individu, París, Seuil, 2013, 103 p. Escribe, en efecto: "El individuo representa una formidable oportunidad para que los partidos de izquierda, siempre que sepan definir una política del individuo emancipado, adquirida con el propósito del Estado de Bienestar, dirigido hacia la realización individual y colectiva; una sociedad en la que todo el mundo estaría reconocido y tratado como un individuo, pero donde todo el mundo, también, estaría protegido y apoyado en su potencial vulnerabilidad. En otras palabras, es inventar una sociedad de individuos. Para lograr esto, el libro aboga por una política de la persona sobre la base de la modernidad de apoyo." (El énfasis aparece en el texto).

12 La autora se inspira en varios trabajos de sociología. En ese sentido, su ensayo traza un puente entre las disciplinas (filosofía y sociología) y requiere de decisiones que promuevan una política de nuevo género que considere a los individuos (sus individualidades) sin caer en la trampa del individualismo.

13 Brugère, Fabienne, La politique de l'individu, op.cit., p.62. "[...] el individuo no se mantiene solo en sociedad. Una política del individuo preocupado por la igualdad, que apunta al bienestar de todos, debe poder organizar los apoyos que, al mismo tiempo, otorgan a todo el mundo las capacidades y las posibilidades de actuar, fuera de estatutos y de particiones; y no olvidan a los individuos más vulnerables, estos 'individuos negativos' que constituyen la prueba de carencia, de la exclusión o de la invisibilidad." 
nueva configuración de las relaciones humanas en un mundo inestable. Su aproximación metodológica difiere, lógicamente, de la propuesta por los filósofos. Como bien lo subraya Marie Garrau:

La catégorie de vulnérabilité est en effet devenue prégnante dans le vocabulaire de la sociologie contemporaine. Ses usages sociologiques sont différents de ceux qui prévalent en philosophie pour plusieurs raisons: quand elle est utilisée par les sociologues, la catégorie de vulnérabilité n'est pas utilisée comme une catégorie anthropologique permettant de décrire une structure d'existence commune et universellement partagée; elle est plutôt mobilisée comme une catégorie analytique et critique, permettant de cerner les effets négatifs produits sur les sujets sociaux par certaines formes d'organisation sociale à un moment historique donné. Par conséquent, les vulnérabilités mises en évidence par les sociologues sont des vulnérabilités sociales et historiques, car dépendantes d'une configuration sociale et historique particulière. La catégorie de vulnérabilité n'a donc pas dans leurs travaux la prétention à la généralité qui caractérisent ses usages en philosophie. ${ }^{14}$

Las vulnerabilidades desarrolladas por los sociólogos son, en efecto, múltiples, pues cada una de ellas tiene un entorno particu-

14 Garrau, Marie, "Regards croisés sur la vulnérabilité. 'Anthropologie conjonctive' et épistémologie du dialogue", en Tracés. Revue de Sciences humaines, 2013, p. 154. "La categoría de vulnerabilidad, en efecto, se ha estado gestando en el vocabulario de la sociología contemporánea. Por muchas razones, los usos sociológicos son diferentes de los que se utilizan en la filosofía: cuando es utilizada por los sociólogos, la categoría de vulnerabilidad no es utilizada como una categoría antropológica que permite describir una estructura de existencia común y universalmente compartida; se usa, sobretodo, como una categoría analítica y crítica, que permite delimitar los efectos negativos producidos por los sujetos sociales, en ciertas formas de organización social y en un determinado momento. Por consecuencia, las vulnerabilidades evidenciadas por los sociólogos son vulnerabilidades sociales e históricas, porque dependen de una configuración social e histórica particulares. Por tanto, la categoría de vulnerabilidad, en los trabajos de los sociólogos, no tiene la pretensión de la generalidad, que caracterizan los usos en la filosofía." 
lar: el trabajo, ${ }^{15}$ la migración, ${ }^{16}$ la prostitución, ${ }^{17}$ etcétera. Si existen múltiples trabajos que nutren el campo de la sociología más allá del Atlántico (al Norte como al Sur del continente americano) y que abordan además múltiples formas de vulnerabilidad (ambientales o industriales, por ejemplo) la sociología francesa llega un poco más tarde y se concentra, específicamente, en las temáticas sociales. ${ }^{18}$ Las investigaciones de Robert Castel son emblemáticas, respecto de un importante trabajo que tiene por eje la vulnerabilidad social "à la conjonction de la précarité du travail et de la fragilité du lien social"19 en donde lo que el llama la "désaffiliation" tiene un lugar central. Los trabajos de Serge Paugam, ${ }^{20}$ como también los de Marc-Henry Soulet, destacan, en el mismo sentido, una aproximación que tiene como hilo conductor las realidades sociales contemporáneas.

La vulnerabilidad ha llegado tarde al campo del derecho. Los estudios pioneros han sido los de la estadounidense Martha Albertson Fineman que cuestionan la "autonomía" de los individuos evolucionados en un ambiente liberal. Según el modelo de las nuevas corrientes filosóficas, ha también introducido el discurso americano clásico sobre la igualdad, partiendo del postulado según el cual cada

15 Cfr. Castel, Robert, L'insécurité sociale. Qu'est-ce qu'être protégée, París, Seuil, 2003, 96 p.

16 Cfr. Cambrezy, L. y Charbit, Y. (eds.), "Dossier spécial: Migrations internationales et vulnérabilités", en Revue européenne des migrations internationales, vol. 23-n³, 2007.

17 Pueden verse los trabajos de Mathieu, L., "La prostitution, zone de vulnérabilité sociale", en Nouvelles questions féministes, vol. 21, n², 2002, pp. 55-75.

18 Para una presentación de abundamiento sociológico en la materia, vid. SYLVIA Becerra, "Vulnérabilité, risques et environnement: l'itinéraire chaotique d'un paradigme sociologique contemporain", en VertigO, La revue électronique en sciences de l'environnement, vol.12, $\mathrm{n}^{\circ} 1$, mai 2012.

19 Robert Castel, "De l'indigence à l'exclusion, la désaffiliation. Précarité du travail et vulnérabilité relationnelle", en J. Donzelot (dir.), Face à l'exclusion. Le modèle français, París, Editions Esprit, 1991, p. 153.

20 Serge Paugam, Le lien social, París, PUF, 2008, 128 p. 
ser humano es, por esencia, vulnerable. Se trata, ni más ni menos, de una condición humana universal. Por tanto, esta condición no debe ser comprendida exclusivamente en términos negativos (sufrimiento, herida, etcétera.) sino que debe ser vista también como portadora de acciones positivas, tanto de la parte de los individuos entre sí, como con el Estado. Fineman deduce que esto último debe ser más "reactivo": es el concepto del Responsive State que deja transparentar el alcance normativo de su teoría. Si tal discurso puede parecer relativamente banal en el contexto europeo, -donde el intervencionismo estatal ha sido, y permanece todavía, como el sello de la política del Viejo continente- la propuesta aparece como radical en el universo americano, donde la no-intervención del Estado permanece todavía como un dogma difícil de deshacer. Su artículo sobre el "sujeto vulnerable" (Vulnerable Subject), ${ }^{21}$ fue el inicio de numerosas reflexiones y discusiones que desembocarían en una obra colectiva de 2013, -dirigida en colaboración con Ana Grear- donde cada autor analiza, bajo un ángulo particular y bien "situé", la teoría de la vulnerabilidad tal como la propuso M. Fineman. ${ }^{22}$

Los juristas, ${ }^{23}$ principalmente en el campo del estudio de los derechos humanos, han terminado por considerar la medida de la importancia de este nuevo concepto; aunque los estudios jurídicos se encuentran todavía en estado embrionario (sobre todo si se les compara con los trabajos filosóficos, sociológicos, médicos científicos en la materia). Lo que es cierto, es que los actores u operadores del derecho -y no solamente los representantes del campo académi$\mathrm{Co}^{-}$, se han apropiado del concepto de vulnerabilidad. Por ejemplo,

21 Cfr. M. A. Fineman, "The Vulnerable Subject: Anchoring Equality in the Human Condition", en 20 Tale F.L. EF Feminism, 9 (2008-2009).

22 Cfr. M. A. Fineman, A. Grear (dirs.), Vulnerability. Reflections for a New Ethical Foundation for Law and Politics, Burlington, Ashgate, 2013, 240 p.

23 En el universo francófono, principalmente francés, el estudio de Charlotte Denizeau recoge los estudios que se han desarrollado a partir del inicio de la década pasada, sobre la vulnerabilidad. 
las diversas contribuciones de esta obra demuestran que los legisladores y los jueces han incorporado este nuevo campo semántico. ${ }^{24}$ Personas y grupos "vulnerables" han irrumpido en el campo de la protección de los derechos humanos. Los profesionales del derecho han manejado el concepto para entender mejor y comprender situaciones específicas, que lejos de ser inéditas, han aparecido en contextos contemporáneos desvelando nuevas problemáticas.

En este campo jurídico, si el concepto de la vulnerabilidad ha florecido, su conceptualización no ha sido regular. Legisladores y jueces se han contentado (lógicamente respecto de sus funciones respectivas) con utilizarlo, sin más, para enmarcar de mejor manera la acción del Estado. Se asistiría por tanto a un Vulnerability Turn ${ }^{25}$ que, a su vez, permitiría repensar al ser humano, su relación con el Otro y con el mundo que lo rodea y, al mismo tiempo, sentaría la base de un conjunto de nuevas obligaciones impuestas al Estado.

En ese escenario ¿todo sería perfecto?; nada de eso. Si la vulnerabilidad va viento en popa, no significa que no haya sufrido, o sufra, virulentas críticas. En Francia, la obra de la politóloga Hélène Thomas - próxima a un panfleto- da cuenta de la existencia de frecuentes rechazos a esta nueva gramática de la subjetividad contemporánea. ${ }^{26}$ La autora pasa la criba a las ideas de los defensores más emblemáticos de los estudios sobre la vulnerabilidad y rechaza

24 En algunos países, son los legisladores quienes se ubican en el origen de la puesta en órbita del concepto; lo que ha generado, lógicamente, que los jueces intervengan para determinar los límites y las modalidades de la aplicación. Ana Ovejero lo demuestra de maravilla. En una cierta medida, y en otro contexto, el análisis de Edouard Dubout lo demuestra también. En efecto, señala que algunas directivas identifican categorías de personas como "vulnerables", por ejemplo, los que solicitan asilo.

25 Se utiliza la expresión famosa que consagra el cambio de "paradigma" en el análisis del lenguaje (el Linguistic Turn), y recientemente, aquella de Droit Global (Global Turn). Sobre este último fenómeno, cfr. M. Xifaras, "Après les Théories Générales de l'Etat: le Droit global?”, en Fus Politicum, nº 8, 2012.

26 Cfr. Hélène Thomas, Les vulnérables. La démocratie contre les pauvres, 2010, Bellecombe-en-Bauges, Editions du Croquant, 254 p. (Col. Terra). 
su aproximación ligada a la compasión, que discrimina más de lo que une. $^{27}$ De manera menos comprometida, Marc-Henry Soulet, en el marco de esta obra - representante de la generación de sociólogos que se han inclinado sobre este concepto-, no ha dado cuenta, con objetividad, de las principales dificultades que produce un tal concepto. Entre los "señuelos de la vulnerabilidad", como él les llama, el jurista está más interesado, principalmente, por el hecho de que se trata de un concepto de "geometría variable" y, sobretodo, que enfrenta el riesgo de una "reducción ontológica". El peligro de la esencialización es mayor y deriva, además -lógicamente hacia la estigmatización-, elementos ya señalados igualmente por Samantha Besson. ${ }^{28} \mathrm{O}$, si se inclina por la cartografía judicial que se desarrolla de las diversas contribuciones de esta obra, se toma la justa medida de la aproximación basada en categorías y, por tanto, diferenciada del uso de la vulnerabilidad que realizan los jueces. Haciendo justicia a todo tipo de dificultades contemporáneas (socio-económicas y/o familiares, criminales, mafiosas, etcétera,) vividas (por no decir, sufridas) por los seres humanos, enfrentados con sociedades cargadas de inseguridad y, ellas mismas vulnerables, los jueces - pensando en la vulnerabilidad de los migrantes (los que solicitan asilo, principalmente), los mineros, las mujeres, las

27 Hélène ataca violentamente a los sociólogos como Robert Castel y Serge Paugam que ella identifica como los jefes de la línea de la courant précaritaire y les reprocha, precisamente, de enviar a los "frágiles, a los vulnerables, a los inseguros [...] con conmiseración y distancia a su inexistencia social, a la incertidumbre y a la inestabilidad.", ibid, p. 99. No perdona tampoco a Amartya Sen, el economista indio que desarrolla el concepto de las capabilities, de las capacités.

28 Lourdes Peroni y Alexandra Timmer -quienes no ocultan su atracción por una plataforma de análisis de los derechos humanos, donde la vulnerabilidad juega un rol esencial- están, sin embargo, conscientes de los riesgos inherentes a un uso judicial, no dominado, de la vulnerabilidad aprehendida a través de los "grupos" (Vulnerable group concept). Dan cuenta de los riesgos de la esencialización, de estigmatización y de paternalismo. Cfr. "Vulnerable groups: The Promise of an Emerging Concept in European Human Rights Convetion Law", en International Journal of Constitutional Law (I. Con), vol. 11, n4, 2013, pp.1056-1085. 
comunidades indígenas, las minorías (por ejemplo, los roms-gitanos-), los detenidos, las personas con discapacidad, los portadores del sida, etcétera... no aprehenden sino lo específico, lo particular, y con ello reducen al individuo a una sola parte de eso que están resaltando, que son sus debilidades. La vulnerabilidad consubstancial al ser humano, la vulnerabilidad - condición humana universal- señalada por la mayoría de los filósofos y de algunos juristas, como Martha Finnema ${ }^{29}$ no ha sido propuesta por los jueces. Solamente lo son las vulnerabilidades evidentes. Se podría decir aquí que los jueces actúan según el modelo de los sociólogos: son los testigos del pathos; porque ninguna valorización positiva de la vulnerabilidad está concedida a los individuos por la jurisprudencia. Respecto de la relación con el Otro, la relación de los individuos con la sociedad (inherente al concepto de la vulnerabilidad) es siempre negativa. En este sentido, ¿no sería oportuno que los jueces abordaran las situaciones desde lo universal antes que desde lo particular o concreto (recordando lo esencial; esto es, que el ser humano es en sí mismo vulnerable)? ${ }^{30} \mathrm{~A}$ partir de ahí, ¿no habría una perspectiva de análisis mejor ubicada para valorizar los contextos de vulnerabilidad, sobre todo respecto de las personas y/o grupos vulnerables?

No sería tanto si los detenidos, las mujeres, las minorías podrían ser calificados de vulnerables, sino los contextos de detención, de la dominación masculina, de la dominación del modelo mayoritario, de la migración (y de otros tránsitos de seres humanos); esos con-

29 Así, Martha Nussbaum desarrolla dos tipos de vulnerabilidades, la vulnerabilidad "constitutiva" (aquella relativa a las capacidades naturales de todo ser humano y que necesita de una intervención política) y las vulnerabilidades segundas (que se manifiestan en una incapacidad de ser y de hacer cuando la intervención política es defectuosa.

зо Samamtha Besson considera que sobre la vulnerabilidad-consubstancial al ser humano, la Corte no tiene que justificarse. En contra, sobre las vulnerabilidades particulares, la Corte no ha desarrollado ninguna argumentación, y debería hacerlo. 
textos serían el curso para adaptar la situación vivida por ciertas personas, en un momento dado y en un contextos determinado.

Si la jurisprudencia de la Corte Europea de Derechos Humanos recuerda frecuentemente, alrededor de una frase, un contexto original (principalmente histórico) de la vulnerabilidad de personas y/o de ciertos grupos, el incidente no es absolutamente suficiente. Por otro lado, la jurisprudencia interamericana, que siempre ha otorgado un lugar central a los contexto (históricos, sociológicos, culturales, etcétera), es mucho más satisfactoria; y debería constituir un aguijón para que el entorno de la vulnerabilidad sea explicado y no apenas visto superficialmente.

Más allá de esta tensión entre 'vulnerabilidad - consubstancial al ser humano' y 'vulnerabilidades particulares de algunas personas y grupos de personas', hay otra tensión señalada por la jurisprudencia: la cuestión normativa que implica el uso de la vulnerabilidad. El incremento (que parece incontrolable) de las obligaciones positivas reforzadas, de las cuales deben ocuparse los Estados, no se ha desarrollado sin que genere problema. Además está el hecho de que ese incremento se trata de una creación pretoriana, susceptible de producir la ira de los Estados (para utilizar un lenguaje lleno de eufemismo), y que podría volver "vulnerable" a la propia Corte Europea (en detrimento de su legitimación). El diseño, el alcance, la exigibilidad de las obligaciones positivas reforzadas son puntos cruciales y complejos que necesitan una política jurisprudencial consolidada. El objetivo parece evidente : reforzando las obligaciones con carga a los Estados respecto de personas o grupos de personas, los jueces consentirían rectificar la multitud de injusticias sufridas, y de esa manera, se podría llegar a la igualdad substancial. ${ }^{31}$

¿Esto equivale a decir que los conceptos tradicionales para conseguirlo (como aquellos de la discriminación indirecta) no son suficientes, o ya no son suficientes? ¿cual sería el valor agregado

31 Sobre la cuestión de la igualdad substancial, el caso de Francia se trata aparte. Al respecto, puede revisarse la comunicación de Charlotte Denizeau. 
de la vulnerabilidad ? ¿No se enturbiaría inútilmente el paisaje? Sobre todo, ¿cómo asegurar la aceptabilidad de las obligaciones reforzadas para que el "fardo" o la carga, no sea considerada por las autoridades como desmedida, y las exigencias correspondientes al interés general sean igualmente preservadas y aseguradas? Muchas otras preguntas como estas conviene que se sigan explorando en el futuro.

Se trata pues de las dos tensiones más fuertes (hay evidentemente otras tantas) generadas por el concepto de la vulnerabilidad, que exigen una reflexión profunda para intentar superarlas o, mejor, conciliarlas. Marie Garrau, en su artículo publicado en la Revue de Sciences Humaines, apuntaba la necesidad de un diálogo disciplinario entre filósofos y sociólogos, a fín de que pudieran enriquecer sus diferentes aproximaciones metodológicas; el diálogo, desde mi perspectiva, debe expandirse hasta que los juristas encuentren su lugar. Esto necesita de miradas cruzadas y condescendientes unas de otras; de una inmersión de cada uno (situación que, desde luego, no es simple), para que, a fin de cuentas, la vulnerabilidad no sea relegada (fustigada) a la categoría de los conceptos escurridizos; que dejarían únicamente su lugar a lo miserable, a la compasión marcada por la mirada con desdén y desprecio, y a la bien o mal desarrollada estigmatización, donde todos los Diferentes se encuentran una vez más, y entonces, de vuelta a las primeras logias de ostracismo de todo tipo. 